\title{
Statistics of charge transfer through impurities in strongly cor- related 1D metals
}

\author{
A. Komnik ${ }^{1,2}$, B. Trauzettel ${ }^{3}$, and U. Weiss ${ }^{4}$ \\ ${ }^{1}$ Institut für Theoretische Physik, Universität Heidelberg, Philosophenweg 19, D-69120 Heidelberg, Ger- \\ many \\ ${ }^{2}$ Physikalisches Institut, Universität Freiburg, Hermann-Herder-Str. 3, D-79104 Freiburg, Germany \\ ${ }^{3}$ Departement Physik und Astronomie, Universität Basel, Klingelbergstr. 82, CH-4056 Basel, Switzerland \\ ${ }^{4}$ Institut für Theoretische Physik, Universität Stuttgart, Pfaffenwaldring 57, D-70550 Stuttgart, Germany
}

\begin{abstract}
Key words Tomonaga-Luttinger liquids, full counting statistics, quantum impurity problem, fractional quantum Hall effect

PACS 71.10.Pm, 73.43.Cd, 73.50.Td

The article is dedicated to Hermann Grabert on the occasion of his 60th birthday.

We review recent advances in the field of full counting statistics (FCS) of charge transfer through impurities imbedded into strongly correlated one-dimensional metallic systems, modelled by Tomonaga-Luttinger liquids (TLLs). We concentrate on the exact analytic solutions for the cumulant generating function (CGF), which became available recently and apply these methods in order to obtain the FCS of a non-trivial contact between two crossed TLL.
\end{abstract}

\section{Introduction}

The relative abundance of exactly solvable models makes the one-dimensional (1D) systems a unique testing ground for new concepts in condensed matter physics. However, during the last two decades the purely academical purpose of 1D studies transformed into an applied science as such extremely reliable 1D metallic materials as single wall carbon nanotubes (SWNTs) became widespread in laboratories [1]. The surging interest in low-dimensional materials is motivated by the rapidly shrinking lateral sizes of the microelectronic circuitry, which is soon expected to arrive at device dimensions at which any wire is truly one-dimensional from the point of view of current carrying electrons.

The most fundamental difference between the 1D metals and their higher-dimensional counterparts is the role of interactions. While (at least in clean systems) the full perturbative expansion in correlation strength is controllable and even convergent in 3D and leads only to insignificant renormalization of parameters (Landau's Fermi liquid theory), it diverges for one-dimensional systems. This is a clear indication of a new universality class - the so-called Tomonaga-Luttinger liquid (TLL). In its simplest form it has first been discussed by Tomonaga in [2]. A slight generalization was offered by Luttinger [3]. Finally, an extremely convenient field theoretical reformulation has been presented by Haldane [4].

It turns out, that whatever the precise form of the interaction potential, the generic correlation term in the TLL universality class is the short ranged $\delta$-shaped interaction. This and the reduced dimensionality allows one to diagonalize the corresponding Hamiltonian with minimal effort just by a canonical transformation. The physical properties of such systems are very interesting. For instance, there is an algebraic singularity in the single particle density of states around the Fermi energy $E_{F}$ due to a formation of highly correlated collective state. The exponent of the corresponding power law is related to the interaction constant. This effect is reflected in the transport properties of such 1D systems contacted by bulk 3D electrodes - the 
differential conductance is a power law of the applied voltage or temperature whatever is larger [5, 6]. This has been seen in a number of experiments as a zero-bias anomaly [7, 8].

The existence of exact solutions makes the correlated 1D metals extremely interesting for the emerging field of full counting statistics (FCS) [9]. The number of non-interacting electrons which are transferred through a structureless scatterer during a certain waiting time is expected to obey the binomial distribution [10]. It is unlikely that it is still valid as soon as the interaction potential among electrons becomes nonzero. In fact, it is not even clear that under such conditions genuine physical electrons are involved in the transport processes. For instance, in fractional quantum Hall $(\mathrm{FQH})$ samples the currents are believed to be carried by fractionally charged Laughlin quasiparticles which have been observed in noise experiments [11, 12]. 1] Therefore the study of such systems would give important insights into the non-equilibrium properties of strongly correlated electronic systems.

Here we would like to review the developments in the field of FCS of such strongly correlated 1D systems. We start with a brief description of the generic setup, whose transport characteristics one is usually interested in in Section 2 After that, in Section 3, we present an exact analytic solution for the charge transfer statistics, which is possible at one special interaction strength. A generic way to solve the problem at arbitrary interaction strength is discussed in the subsequent Section 4 Finally, as an application of the developed formalism we present some new results for the FCS of a contact between two TLLs in Section 5 .

\section{Impurity in a Tomonaga-Luttinger liquid}

The starting point are the free 1D electrons with a linearized dispersion relation. As a result, the kinetic part describes two different fermion species: the right/left-movers,

$$
H_{0}=i v_{F} \int d x\left[R^{\dagger}(x) \partial_{x} R(x)-L^{\dagger}(x) \partial_{x} L(x)\right]
$$

where $R(x)$ and $L(x)$ are the corresponding field operators, $\hbar=1$, and $v_{F}$ is the Fermi velocity. As one is mostly interested in the system's properties in the universality region which is essentially a low-energy regime, the linearization of the dispersion is unproblematic. Such electron segregation is even natural in FQH systems, where the R/L moving branches are spatially separated from each other and are not even allowed to interact in any way. The generic interaction of the Luttinger liquid universality class turns out to be extremely short ranged and effectively given by a $\delta$-shaped potential, the corresponding Hamiltonian contribution being

$$
H_{I}=U \int d x d y \rho(x) \delta(x-y) \rho(y),
$$

where $U$ is the interaction strength and $\rho(x)$ is the full particle density operator. It turns out that the fastest way to a diagonal Hamiltonian leads through the bosonization representation, where both the kinetic as well as the interaction terms have similar shapes and can be merged by a mere rotation. In this way one obtains

$$
H_{B}=\frac{1}{4 \pi g} \int d x\left[\left(\partial_{x} \phi_{R}\right)^{2}+\left(\partial_{x} \phi_{L}\right)^{2}\right]
$$

where $\phi_{R, L}(x)$ are the bosonic fields which can be directly related to the current density operators $j_{R / L}(x)=$ $\mp \partial_{x} \phi_{R / L}(x)$. Remarkably, the interaction strength is now reworked into the dimensionless parameter $g=1 / \sqrt{1+U /\left(\pi v_{F}\right)}$, which in the case of an FQH bar is obtained differently to be equal to the filling

\footnotetext{
${ }^{1}$ It has been shown in Ref. [13] that the analysis of the data in Ref. [11] is indeed compatible with the TLL theory of FQH edges states.
} 
factor. To come that far it is usually not necessary to know the precise form of the relation between the phase fields and the original physical fermions - the bosonization identity. However, it is necessary as soon as one needs to generate the bosonic form of the interedge tunnelling operator $\sim R^{\dagger}(0) L(0)+L^{\dagger}(0) R(0)$. Fortunately, in the continuum limit this relation reduces up to an overall numerical prefactor to a rather simple prescription $R / L(0) \rightarrow \exp \left[i \phi_{R / L}(0)\right][14,15]$. As at least in the FQHE set-up the forward scattering can clearly be dropped from the outset, the full Hamiltonian then reads $H=H_{B}+H_{B S}$, where the back scattering (in the case of chiral TLL) and interedge tunnelling (in the case of a FQH device) contribution is given by

$$
H_{B S}=\lambda_{B S} \cos \left[\phi_{L}(0)-\phi_{R}(0)\right] .
$$

$\lambda_{B S}$ is the (bare) back scattering amplitude. $H$ is equivalent to the so-called boundary sine-Gordon (BSG) Hamiltonian. From the renormalization group point of view it is known to possess two fixed points: an infrared (low energy) and ultraviolet (high energy) ones. In the infrared limit, which is the one we are interested in, the system becomes strongly coupled for $0<g<1$ (repulsive interactions) and $\lambda_{B S}$ flows to infinity. In the opposite situation $g>1$ (attractive interactions) $\lambda_{B S}$ is irrelevant, we do not consider this situation in the rest of the paper.

\section{Analytic solution via refermionization}

As has been realized by Guinea in another context, the sine-Gordon Hamiltonian is simply diagonalizable not only at the trivial point $g=1$, but also at $g=1 / 2$ as well [16]. Indeed, $\exp \left[i \phi_{L / R}(x)\right]$ trivially satisfies the original fermionic anticommutation relation at $g=1$. On the other hand, setting $g=1 / 2$ one finds that the construction $\exp \left\{i\left[\phi_{R}(x)-\phi_{L}(-x)\right]\right\}$ supplemented by appropriate prefactors is subject to fermionic statistics as well. In this section, we review the results of the FCS of a TLL with a single impurity at the specific interaction parameter $g=1 / 2$. The results apply as well to the FCS of the FQH interedge tunnelling as the problems are isomorph.

In the theory of FCS, the quantity of interest is the generating function $\chi(\lambda)$, which is the Fourier transform of the probability distribution $P(Q)$ of the charge $Q$ crossing the impurity during time $\tau, \chi(\lambda)=$ $\sum_{Q} e^{i \lambda Q} P(Q)$. The function $\chi(\lambda)$ generates moments of the charge $Q_{\tau}=\int_{0}^{\tau} d t I(t)$ transferred during time $\tau$,

$$
\chi(\lambda)=\sum_{k} \frac{(i \lambda)^{k}}{k !}\left\langle Q_{\tau}^{k}\right\rangle=\exp \left[\tau \sum_{k} \frac{(i \lambda)^{k}}{k !}\left\langle\delta^{k} Q\right\rangle\right]
$$

where $\tau\left\langle\delta^{k} Q\right\rangle$ is the $k$ th cumulant of the distribution and $I(t)$ is the time-dependent current through the scattering region. The most powerful tool to calculate the resulting $\chi$ is the Keldysh formalism. It has been shown in Ref. [17] that the generating function for the single impurity problem at $g=1 / 2$ may be written as

$$
\chi(\lambda)=\chi_{0}(\lambda)\left\langle\mathcal{T}_{ \pm} e^{i \int_{0}^{\tau} d t H^{-}(t)} e^{-i \int_{0}^{\tau} d t H^{+}(t)}\right\rangle
$$

with time-dependent Hamiltonians

$$
H^{ \pm}(t)=H_{B}+\int d x 2 \lambda_{B S} \delta(x) \cos \left\{2\left[\phi_{L}(0)-\phi_{R}(0)\right] \mp \frac{1}{4} \lambda-\frac{1}{2} e \tilde{V} t\right\},
$$

where $\mathcal{T}_{ \pm}$orders operators along the Keldysh contour and

$$
\chi_{0}(\lambda)=\exp \left[\tau \frac{e^{2}}{4 \pi}\left(i V \lambda-T \lambda^{2}\right)\right]
$$

\footnotetext{
2 Of course, in case of the conventional TLL the forward scattering is always present. It can easily be taken care of by a mere phase shift of the bosonic phases though.
} 
is the generating function in the absence of backscattering. Furthermore, $\tilde{V}=V+2 i \lambda T$, where $V$ is the applied voltage (that drops at the impurity site), and $T$ is the temperature. If we follow Matveev [18], we can transform the Hamiltonian (5) in an elegant way and express it in terms of a new fermion. This makes the calculation of the generating function a straightforward exercise with the resulting expression

$$
\begin{aligned}
\ln \chi(\lambda) & =\ln \chi_{0}(\lambda) \\
& +\tau \int_{0}^{\infty} \frac{d \omega}{2 \pi} \ln \left\{1+\frac{T_{B}^{2}}{\omega^{2}+T_{B}^{2}}\left[\left(e^{-i \lambda}-1\right) \tilde{f}^{+}\left(1-\tilde{f}^{-}\right)+\left(e^{i \lambda}-1\right) \tilde{f}^{-}\left(1-\tilde{f}^{+}\right)\right]\right\}
\end{aligned}
$$

with $\tilde{f}^{+}=\tilde{f}(\omega)=\{\exp [(\omega-e \tilde{V} / 2) / T]+1\}^{-1}, \tilde{f}^{-}(\omega)=1-\tilde{f}(-\omega)$, and $T_{B}=a \lambda_{B S}^{2} / 2 v_{F}\left(a^{-1}\right.$ is the high-energy cutoff of the TLL theory. $a$ is of the order of the lattice spacing of the underlying lattice model.). It has been shown in App. C of Ref. [19] that Eq. (7) can be brought into the more familiar form

$$
\ln \chi(\lambda)=\tau \int_{0}^{\infty} \frac{d \omega}{2 \pi} \ln \left\{1+\frac{\omega^{2}}{\omega^{2}+T_{B}^{2}}\left[\left(e^{i \lambda}-1\right) f^{+}\left(1-f^{-}\right)+\left(e^{-i \lambda}-1\right) f^{-}\left(1-f^{+}\right)\right]\right\}
$$

with $f^{+}=f(\omega)=\{\exp [(\omega-e V / 2) / T]+1\}^{-1}$ and $f^{-}(\omega)=1-f(-\omega)$. The two equivalent ways of writing the generating function Eqs. (7) and (8) are dual to each other. The reason is that Eq. (7) employs a formulation of the generating function using the energy-dependent reflection coefficient $\mathcal{R}(\omega)=T_{B}^{2} /\left(\omega^{2}+T_{B}^{2}\right)$ whereas Eq. (8) employs a formulation of the generating function using the energy-dependent transmission coefficient $\mathcal{T}(\omega)=\omega^{2} /\left(\omega^{2}+T_{B}^{2}\right)$. In Ref. [17], the first three cumulants have been calculated in terms of special functions for arbitrary transmission, temperature, and applied bias. They read $\left(G_{0}=e^{2} / h\right.$ is the conductance quantum)

$$
\begin{aligned}
\langle\delta Q\rangle & =\frac{1}{2} G_{0} V\left[1-\frac{2 T_{B}}{e V} \operatorname{Im} \psi\left(\frac{1}{2}+\frac{2 T_{B}+i e V}{4 \pi T}\right)\right], \\
\left\langle\delta^{2} Q\right\rangle & =2 T \frac{d\langle\delta Q\rangle}{d V}-\frac{e}{2} T_{B} \operatorname{coth}\left(\frac{e V}{2 T}\right) \frac{d\langle\delta Q\rangle}{d T_{B}}+T T_{B} \frac{d^{2}\langle\delta Q\rangle}{d V d T_{B}}, \\
\left\langle\delta^{3} Q\right\rangle & =\frac{1}{2} T T_{B} \frac{d^{2}\left\langle\delta^{2} Q\right\rangle}{d V d T_{B}}-\frac{e}{2} T_{B} \operatorname{coth}\left(\frac{e V}{2 T}\right) \frac{d\left\langle\delta^{2} Q\right\rangle}{d T_{B}}+2 T \frac{d\left\langle\delta^{2} Q\right\rangle}{d V} \\
& +\frac{e}{2} T T_{B} \operatorname{coth}\left(\frac{e V}{2 T}\right) \frac{d^{2}\langle\delta Q\rangle}{d V d T_{B}}+\frac{e^{2}}{4} T_{B} \sinh ^{-2}\left(\frac{e V}{2 T}\right) \frac{d\langle\delta Q\rangle}{d T_{B}},
\end{aligned}
$$

where $\psi$ is the digamma function. In the zero temperature limit, Eqs. (9) - 111) obey the relations (16) between the higher order cumulants and $\langle\delta Q\rangle$ derived earlier in Ref. [25].

\section{Thermodynamic Bethe ansatz solution at arbitrary interaction}

As has already been mentioned above, the system can be mapped onto the sine-Gordon model by a folding and a subsequent transformation to the even-odd basis [20, 21, 22]. After that procedure the excitations in the system are easily identifiable by means of the Bethe ansatz technique: at integer $1 / \mathrm{g}$ there are current carrying charged kinks-antikinks, which we from now on denote by subscripts \pm , and $j=1 \ldots 1 / g-2$ neutral (charge zero) breathers, which are bound states of kinks and antikinks. Both excitation types are masseless and possess simple dispersion relations in terms of rapidity $\theta: e_{ \pm}=e^{\theta} / 2$ and $e_{j}=\sin [\pi j / 2(1 / g-1)] e^{\theta}$. It is important to realise that these excitations are not related in any simple way to the original electrons. Nevertheless, only (anti)kinks are scattered on the boundary and with a known quite simple scattering matrix $S_{i j}$ given in Ref. [20]. For the latter we shall only need the resulting effective "transmission coefficient" of the (anti)kinks through the boundary,

$$
\mathcal{T}(\theta)=\left|S_{++}(\theta)\right|^{2}=\left|S_{--}(\theta)\right|^{2}=\frac{1}{1+e^{2(1-1 / g)\left(\theta-\theta_{B S}\right)}},
$$

\footnotetext{
${ }^{3}$ We have to keep in mind that all energy scales should be much lower than the high-energy cutoff of the model $1 / a$.
} 
where $\theta_{B S}$ is the effective rapidity corresponding to the back scattering amplitude $\lambda_{B S}$. In the noninteracting case the knowledge of the impurity transmission and of the energy distribution function of the incoming particles (essentially Fermi distributions) would be sufficient for construction of not only the non-linear $I-V$ but of the CGF as well [10]. This is different in the present case as not only are the quasiparticles distributed in a completely different way, the distribution functions of the (anti)kinks [ $\eta_{\mp}(k), k$ being the particle momenta] at different energies (or rapidities) are correlated due to interactions in the bulk. However, that does not matter in the expression for the current through the system. Just as in the non-interacting situation the corresponding expression only involves linear combinations of Fermi distributions and it is natural that no higher correlations of population probabilities emerge even in the interacting case. In fact, as is shown in [21, 22, 23], one obtains for the current

$$
I=I\left(V, T_{B S}\right)=\frac{1}{L} \sum_{k} \mathcal{T}(k)\left(\eta_{+}-\eta_{-}\right)=\int d \theta \mathcal{T}(\theta)\left[\bar{P}_{+}(\theta)-\bar{P}_{-}(\theta)\right]
$$

where $\bar{P}_{ \pm}(\theta)$ is the expectation value of the density of the occupied states, $T_{B S}$ is the temperature (energy) scale associated with $\lambda_{B S}$ and $L$ is the system length which is sent to infinity in the continuum limit (s. below). This situation changes completely as soon as one goes over to the noise properties of the system. Here even in the non-interacting case products of Fermi distribution functions emerge. Thus one has to expect some more involved bilinear products of $\eta_{ \pm}$at different momenta to enter the expression. This indeed has been shown in [23]. The physical interpretation of the emerging picture is then very appealing: the transport is mediated by one by one elastic scattering of (anti)kinks off the boundary with the known scattering matrix. If only one channel with particles with momenta $k$ were involved the corresponding momentum generating function would be given by

$$
\chi_{k}(\lambda)=\left\{1+\mathcal{T}(k)\left[\eta_{+}\left(1-\eta_{-}\right)\left(e^{-i \lambda}-1\right)+\eta_{-}\left(1-\eta_{+}\right)\left(e^{i \lambda}-1\right)\right]\right\}^{\tau / L} .
$$

$\tau$ is again the waiting time and $1 / L$ corresponds to the spectral weight of the single $k$-channel. It can easily be shown that for transport in multiple channels (at different energies) the corresponding momentum generating function is a product of single channel contributions $\hat{\chi}(\lambda)=\prod_{k} \chi_{k}(\lambda)$. The role of the breathers in the bulk however, is to entangle the single-particle energy distribution functions of the (anti)kinks, hence the previous formula has to be averaged over all possible system configurations. Therefore we arrive at the following total CGF,

$$
\ln \chi(\lambda)=\ln \langle\hat{\chi}(\lambda)\rangle=\ln \left\langle\prod_{k}\left\{1+\mathcal{T}(k)\left[\left(e^{i \lambda}-1\right) \eta_{-}\left(1-\eta_{+}\right)+\left(e^{-i \lambda}-1\right) \eta_{+}\left(1-\eta_{-}\right)\right]\right\}^{\tau / L}\right\rangle
$$

which was suggested in [24].

The exact results for $g=1 / 2$ follow from this ansatz quite naturally. First of all under these conditions there are no breathers in the bulk and the (anti)kinks are free fermions. That is why they fill the momentum space like ordinary electrons and are subject to Fermi distributions $\eta_{ \pm}(k)=n_{L / R}(k)=n_{F}(k \mp V / 2)$. Since the quasiparticles are now non-interacting, the averaging in $(15)$ is superfluous and must be dropped. Furthermore, the transmission coefficient (12) is given by $\mathcal{T}(k)=k^{2} /\left(k^{2}+\lambda_{B S}^{2}\right)$ as $k=D e^{\theta}$ and $\lambda_{B S}=D e^{\theta_{B S}}, D$ being a cut-off which falls out of any observable. Combining everything we immediately obtain the result (8).

Another important limiting case is the zero temperature regime. Here all correlations among the distributions $\eta_{ \pm}(k)$ vanish and all irreducible momenta turn out to be given by [25]

$$
\left\langle\delta^{n} Q\right\rangle=\frac{\tau}{L} \sum_{k} F_{n}(k)\left(\eta_{+}-\eta_{-}\right)=\tau \int d \theta F_{n}(\theta)\left[\bar{P}_{+}(\theta)-\bar{P}_{-}(\theta)\right]
$$


where $F_{1}(\theta)=\mathcal{T}(\theta), F_{2}(\theta)=\mathcal{T}(\theta)[1-\mathcal{T}(\theta)], F_{3}(\theta)=\mathcal{T}(\theta)[1-\mathcal{T}(\theta)][1-2 \mathcal{T}(\theta)]$ etc. A following very convenient relation between different $F_{n}$-functions can be verified,

$$
F_{n}(\theta)=\frac{\partial^{n-1}}{\left(\partial \widetilde{\theta}_{B S}\right)^{n-1}} F_{1}(\theta)
$$

with $\widetilde{\theta}_{B S}=-2(1-g) g \theta_{B S}$, which helps to reduce every single cumulant to a calculation of the current derivative with respect to impurity rapidity,

$$
\left\langle\delta^{n} Q\right\rangle=\frac{\partial^{n-1}}{\left(\partial \widetilde{\theta}_{B S}\right)^{n-1}}\langle\delta Q\rangle .
$$

Summing up all cumulants into the generating functional one obtains

$$
\frac{\partial}{\left(\partial \widetilde{\theta}_{B S}\right)} \ln \chi(\lambda)=\sum_{m=1}^{\infty} \frac{(i \lambda)^{m}}{m} \frac{\partial^{m}}{\left(\partial \widetilde{\theta}_{B S}\right)^{m}} I\left(V, \widetilde{\theta}_{B S}\right)=I\left(V, \widetilde{\theta}_{B S}+i \lambda\right)-I\left(V, \widetilde{\theta}_{B S}\right) .
$$

Although in general no closed analytic expressions for the current exist, the expansions for strong and weak back scattering turn out to be very useful. There are clear physical pictures in these limits [25]. When the system is in vicinity of the weak coupling point, the true ground state is that of two completely disconnected systems. It is evident that then only physical electrons with integer charge may tunnel between the subsystems. On the other hand, in the strongly coupled system a collective state between the edges is formed, which in the case of a FQH bar has fractionally charged Laughlin quasiparticles for elementary excitations.

For strong backscattering or weak tunneling, $V / T_{B S}^{\prime} \ll 1$, where $T_{B}^{\prime}$ is defined by

$$
T_{B}^{\prime} / T_{B}=(2 \sqrt{\pi} / g) \Gamma\{1 / 2+g /[2(1-g)]\} / \Gamma\{g /[2(g-1)]\},
$$

one obtains

$$
\ln \chi(\lambda)=\tau \sum_{m=1}^{\infty}\left(e^{i \lambda m}-1\right) k_{m}
$$

where

$$
k_{m}=\frac{1}{m} a_{m}(1 / g) G_{0} V\left(V / T_{B S}^{\prime}\right)^{2 m(1 / g-1)}
$$

with [21]

$$
a_{m}(g)=(-1)^{m+1} \frac{\Gamma(3 / 2) \Gamma(m g)}{\Gamma(m) \Gamma[3 / 2+m(g-1)]} .
$$

Observe that the counting field $\lambda$ enters the expression (18) with an integer prefactor $m 4$ The physical meaning of the expression (18) is quite illuminating. Suppose that $k_{m}$ is the probability per unit time to transfer a particle of charge $m$ through the impurity barrier. Then the charge transferred in the time interval $\tau$ is the result of a Poisson process for particles of charge one crossing the barrier, contributing a current $I_{1}=k_{1}$, plus a Poisson process for particles of charge two contributing a current $I_{2}=2 k_{2}$, etc. The logarithm of the Fourier transform of the probability distribution of all these Poisson processes would then be the expression (18). The only subtle point now is that the signs of the rates $k_{m}$ are not quite right since

\footnotetext{
${ }^{4}$ It has been argued in [26] that in the general case the CGF contains prefactors $\exp \left( \pm i \lambda\left|e^{*} / e\right|\right)$, where $e^{*}$ is the charge of current carrying excitations.
} 
a classical process would require that all rates are positive. Certainly, the $m=1$-term is indeed a Poisson process for the tunneling of single electrons, but the joint tunneling of pairs of electrons (and of multiples thereof) $m=2,4, \cdots$ comes with the different sign, which is an effect of quantum interference.

In the opposite limit of weak backscattering, the expression for $\ln \chi(\lambda)$ reads

$$
\ln \chi(\lambda)=\tau\left[i \lambda g G_{0} V+\sum_{m=1}^{\infty}\left(e^{-i \lambda g m}-1\right) \widetilde{k}_{m}\right]
$$

where

$$
\widetilde{k}_{m}=\frac{1}{m} a_{m}(g) g G_{0} V\left(V / T_{B S}^{\prime}\right)^{2 m(g-1)} .
$$

This form is quite similar to (18), but there are subtle differences. The first term in the expression (21) represents the current of fractionally charged quasiparticles in the absence of the barrier. The exponential factor $e^{-i \lambda g m}$ indicates that now we have tunneling of quasiparticles of charge $g$ and of multiples thereof, and the minus sign in the exponent means that the tunneling diminishes the current instead of building it up as in the strong-backscattering regime. The sign of the tunneling rate $\widetilde{k}_{m}$ is now that of $\cos (m \pi g)$. Therefore, the perception of clusters of quasiparticles with fractional charge $g$ tunneling independently with a classical Poisson process is quite appropriate when $g$ is small. The classical limit is reached as $g$ goes to zero. Then all the rates $\widetilde{k}_{m}$ are positive, but the quantum fluctuations $\left\langle\delta^{n} Q\right\rangle$ with $n>1$ have faded away,

$$
\ln \chi(\lambda)=i \lambda \tau g G_{0} V\left[1-\sum_{m=1}^{\infty} \frac{\Gamma\left(m-\frac{1}{2}\right)}{2 \sqrt{\pi} m !}\left(\frac{T_{B S}^{\prime}}{V}\right)^{2 m}\right]=i \lambda \tau g G_{0} V \sqrt{1-\left(\frac{T_{B S}^{\prime}}{V}\right)^{2}}
$$

These results have been derived relying on the integrable approach. Alternatively, one may return to the bosonic representation, the BSG model (1) with (2). Upon integrating out the Luttinger liquid modes away from the impurity located at $x=0$, one arrives at the Coulomb gas representation of the quantum impurity problem which at zero temperature involves long-range logarithmic interactions. We see from this representation that the Luttinger liquid modes act as an Ohmic thermal reservoir [28]. Reconsidering the cumulant generating function in the Coulomb gas representation with the rigorous nonequilibrium Keldysh or Feynman-Vernon method, one finds confirmation of the expressions (18) - (22) and deeper understanding of the cumulant relation (16) [25, 27].

For arbitrary impurity strength or finite temperatures one has to evaluate (15) with the help of thermodynamic Bethe ansatz technique. The evaluation of $\eta_{i}(k)$ products is rather demanding. In most of the experiments, however, still only the cumulants of lowest orders are measured. In [24] a general procedure for the calculation of cumulants of any order is presented and the third irreducible moment is explicitly calculated.

Interestingly, the leading low-temperature contribution to the zero temperature cumulant generating function can be calculated in analytic form. To this aim, we work in the Coulomb gas representation and perform the low temperature expansion of the charge interaction factor, which is a power series in $T^{2}$. We find that the $T^{2}$-contribution to the cumulant generating function can be universally written in terms of the second derivative of the zero temperature expression with respect to the voltage, whereas higher-order terms are non-universal. In the end we find the CGF for strong backscattering in the form (18) with the rate expressions $k_{m}$ given by

$$
k_{m}(V, T)=\left[1+\frac{\pi^{2}}{3 g} T^{2} \frac{\partial^{2}}{(\partial V)^{2}}\right] k_{m}(V)=\left\{1+\frac{\pi^{2}}{3 g}\left(\frac{2}{g}-2\right) m\left[1+\left(\frac{2}{g}-2\right) m\right] \frac{T^{2}}{V^{2}}\right\} k_{m}(V),
$$


where terms of order $T^{4}$ are disregarded. In the opposite weak-backscattering limit we obtain in generalization of the expression (21)

$$
\ln \chi(\lambda)=\tau\left[\left(i \lambda V-\lambda^{2} T\right) g G_{0}+\sum_{m=1}^{\infty}\left(e^{-i \lambda g m}-1\right) \widetilde{k}_{m}(V, T)\right],
$$

where (modulo terms of order $T^{4}$ )

$$
\widetilde{k}_{m}(V, T)=\left[1+\frac{\pi^{2} g}{3} T^{2} \frac{\partial^{2}}{(\partial g V)^{2}}\right] k_{m}(V)=\left\{1+\frac{\pi^{2}}{3 g}(2 g-2) m[1+(2 g-2) m] \frac{T^{2}}{V^{2}}\right\} \widetilde{k}_{m}(V) .
$$

The $T^{2}$-contribution to the cumulants is a distinctive signature of interacting $1 \mathrm{D}$ electrons. A related phenomenon is the universal $T^{2}$-behavior observed at low temperatures in open quantum systems with Ohmic dissipation, e.g. $T^{2}$-enhancement in macroscopic quantum tunneling [28]. It is also the origin of the universal Wilson ratio, observed e.g. in Kondo systems.

\section{Application: FCS of the crossed TLLs set-up}

It is expected that as the lateral dimension of the microelectronic circuitry becomes smaller, the ultimate nanoscale wiring would become truly one-dimensional from the point of view of current carrying electrons. Indeed, a metallic wire with a thickness of only several $\mathrm{nm}$ can only support electrons propagating as an $s$-wave since the states with higher angular momenta lie so high that even at room temperature they cannot be excited. Thus, in the future nanoelectronics one has strongly interacting quantum wires as one of the basic circuitry elements. The next to a simple wire complexity level is achieved by a point contact of two such conductors. In fact, following the theoretical proposal of [29] a number of successful experiments has been already conducted on SWNT junctions measuring the corresponding non-linear $I-V$ [30, 31]. We expect that noise or even FCS measurement will be conducted on such set-ups in the nearest future.

The most general way of coupling two conductors is not only by a particle exchange via tunnelling, but by an electrostatic interaction as well. As has been discussed in [29], in the true fixed point the inter-wire tunnelling turns out to be completely irrelevant. Moreover, although the density-density Coulomb coupling between the wires consists of a number of terms only one of them with the lowest scaling dimension survives in the fixed point. The effective Hamiltonian is then given by

$$
H=\sum_{j=1,2} H_{B j}\left[\phi_{R / L j}\right]+\lambda_{C} \sin \left[\phi_{L 1}(0)-\phi_{R 1}(0)\right] \sin \left[\phi_{L 2}(0)-\phi_{R 2}(0)\right],
$$

where $H_{B j}\left[\phi_{R / L j}\right]$ is given by (1) for the wire $j=1,2$ and $\lambda_{C}$ is the coupling constant. A very convenient simplification of the problem is achieved by introduction of channel (anti)symmetric variables according to the prescription

$$
\phi_{R / L \pm}=\frac{\phi_{R / L 1} \pm \phi_{R / L 2}}{\sqrt{2}}
$$

In this way the original problem separates into two commuting contributions,

$$
H=\sum_{p= \pm} H_{B p}\left[\phi_{R / L p}\right]+p \frac{\lambda_{C}}{2} \cos \left\{\sqrt{2}\left[\phi_{L p}(0)-\phi_{R p}(0)\right]\right\} .
$$

It turns out that by the above rotation even the non-equilibrium transport problem separates. Not only the voltage applied to the new \pm channels is given by the (anti)symmetric combination of the original $V_{1,2}$,

\footnotetext{
${ }^{5}$ Here the equality of the interaction constants $g_{1}=g_{2}=g$ is assumed, but this requirement is not restrictive in any way and a more general transformation can easily be conceived.
} 
but the physical currents in the 1,2 channels are calculated via prescription $I_{1,2}=\left(I_{+} \pm I_{-}\right) / \sqrt{2}$. In fact, this separation is even more fundamental: it is easily realised that even the FCS should factorize into two independent contributions with counting fields $\lambda_{ \pm}=\left(\lambda_{1} \pm \lambda_{2}\right) / \sqrt{2}$ as

$$
\ln \chi\left(\lambda_{1}, \lambda_{2}\right)=\ln \left[\chi\left(\lambda_{+}, V_{+}\right) \chi\left(\lambda_{-}, V_{-}\right)\right]
$$

where $\chi(\lambda, V)$ is given by (15). Of course, the first order cumulant with respect to either of the channels leads to the non-linear $I-V$ as discussed in [29], provided one employs the "voltage drop" way of coupling to the voltage sources [5].

Now we want to analyse correlations of higher orders. The direct (within one of the channels $j=1,2$ ) noise contribution is trivially found by double derivative,

$$
\left\langle\delta^{2} Q_{j}\right\rangle=(-i)^{2} \frac{\partial^{2}}{\partial \lambda_{j}^{2}} \ln \chi\left(\lambda_{1}, \lambda_{2}\right)=\left(\frac{\partial \lambda_{-}}{\partial \lambda_{i}}\right)^{2} P_{-}+\left(\frac{\partial \lambda_{+}}{\partial \lambda_{i}}\right)^{2} P_{+},
$$

where

$$
P_{ \pm}=-\frac{\chi\left(\lambda_{ \pm}, V_{ \pm}\right) \partial_{ \pm}^{2} \chi\left(\lambda_{ \pm}, V_{ \pm}\right)-\left[\partial_{ \pm} \chi\left(\lambda_{ \pm}, V_{ \pm}\right)\right]^{2}}{\chi^{2}\left(\lambda_{ \pm}, V_{ \pm}\right)}
$$

The corresponding explicit formulas are given in [32]. As $\left(\partial \lambda_{ \pm} / \partial \lambda_{1,2}\right)^{2}=1 / 2$ the noise locking found in [32] follows immediately,

$$
\left\langle\delta^{2} Q_{j}\right\rangle=\left(P_{+}+P_{-}\right) / 2 .
$$

Another interesting quantity is the yet unstudied cross correlation

$$
\begin{aligned}
\left\langle\delta Q_{1} \delta Q_{2}\right\rangle & =(-i)^{2} \frac{\partial^{2}}{\partial \lambda_{1} \partial \lambda_{2}} \ln \chi\left(\lambda_{1}, \lambda_{2}\right)=\left(\frac{\partial \lambda_{-}}{\partial \lambda_{1}}\right)\left(\frac{\partial \lambda_{-}}{\partial \lambda_{2}}\right) P_{-}+\left(\frac{\partial \lambda_{+}}{\partial \lambda_{1}}\right)\left(\frac{\partial \lambda_{+}}{\partial \lambda_{2}}\right) P_{+} \\
& =\left(P_{+}-P_{-}\right) / 2
\end{aligned}
$$

Remarkably, this correlation vanishes completely when there is no net transport in either of the wires. Even more surprising feature is that this kind of cancellation is temperature-independent. Thus the correlations (32) are unaffected by the Johnson-Nyquist noise. This phenomenon along with the effect (31) could be used in future experiments to identify the sophisticated multi-particle state the system is in.

\section{Conclusions}

In most cases the task of FCS calculation in a generic system requires application of nonequilibrium techniques. Usually this is a rather challenging endeavour for strongly correlated systems, where pronounced deviations from the conventional charge transfer statistics are expected. One class of such systems covers interedge tunneling in the fractional quantum Hall bars and impurities imbedded into chiral TomonagaLuttinger liquids. Here the charge of current carrying excitations is different from the elementary charge. This results in a unique FCS, which differs considerably from the noninteracting setups.

Despite the fact, that these systems are integrable with help of the thermodynamic Bethe ansatz, there is no possibility to exploit it directly to address their FCS. Nevertheless, it still can be accessed using a number of shortcuts. One of them is the possibility to map the corresponding boundary sine-Gordon model to a noninteracting fermionic theory via refermionization procedure at some special nontrivial interaction strength. The resulting Hamiltonian turns out to be quadratic and easily diagonalizable with elementary methods making a genuine nonequilibrium evaluation of the FCS a rather simple task.

Another way towards the FCS is offered by the equilibrium Bethe ansatz solution, which is able to identify all possible excitations in the system as well as the corresponding scattering matrices. It turns out 
that for the generic interaction strength there are only two excitations which are responsible for the transport: kinks and antikinks, whose reflections on the boundary correspond to the physical current carrying processes. All other excitations are merely responsible for the rather involved (non-Fermi like) energy distribution functions. The values for the probabilities to occupy (anti)kink states at different momenta $\eta_{ \pm}(k)$ are not mutually correlated as long as the system is at zero temperature. Thus the application of elementary statistical arguments immediately leads to the FCS. The same result could be achieved by a straightforward Coulomb gas expansion method. Interestingly, this approach is also able to supply the leading corrections around the $T=0$ result.

At finite temperatures the distributions $\eta_{ \pm}(k)$ are correlated with each other prohibiting the FCS calculation along this path. The cumulant generating function $\chi(\lambda)$ can be evaluated nonetheless by an ansatz which takes advantage of the fact, that the current transport is mediated by momentum conserving scattering of (anti)kinks off the boundary. Indeed, the FCS can be expressed as a complicated average of a product of $\chi_{k}(\lambda) s$ describing transport in individual channels corresponding to a designated momentum value.

All these techniques are not restricted to the single impurity problems only. They can rather be applied to access the FCS of more complicated junctions and networks of quantum wires in the TLL state. We expect, that this kind of information is especially interesting in view of recent advances in the fields of nanoelectronics, spintronics and quantum computation.

Acknowledgements AK acknowledges the support by the DFG grant KO 2235/2. BT was supported by the Swiss NSF and the NCCR Nanoscience. The authors would like to thank Hubert Saleur and Markus Kindermann for numerous fruitful discussions.

\section{References}

[1] S. Iijima, Nature 354, 56 (1991).

[2] S. Tomonaga, Prog. Theor. Phys. Osaka 5, 349 (1950).

[3] J. M. Luttinger, J. Math. Phys. 4, 1154 (1963).

[4] F. D. M. Haldane, J. Phys. C: Solid State Phys. 14, 2585 (1981).

[5] C. L. Kane and M. P. A. Fisher, Phys. Rev. B 46, 15233 (1992).

[6] A. Furusaki and N. Nagaosa, Phys. Rev. B 47, 4631 (1993).

[7] M. Bockrath et al., Nature 397, 598 (1999).

[8] Z. Yao et al., Nature 402, 273 (1999).

[9] Yu. V. Nazarov, see this volume.

[10] L. S. Levitov and G. B. Lesovik, JETP lett. 58, 230 (1993).

[11] R. de Picciotto et al., Nature 389, 162 (1997).

[12] L. Saminadayar et al., Phys. Rev. Lett. 79, 2526 (1997).

[13] B. Trauzettel, P. Roche, D. C. Glattli, and H. Saleur, Phys. Rev. B 70, 233301 (2004).

[14] D. C. Mattis, J. Math. Phys. 15, 609 (1974).

[15] A. O. Gogolin, A. A. Nersesyan, and A. M. Tsvelick, Bosonization and strongly correlated systems, Cambridge Unviersity Press (1998).

[16] F. Guinea, Phys. Rev. B 32, 7518 (1985); see also U. Weiss et al., Phys. Rev. B 52, 16707 (1995).

[17] M. Kindermann and B. Trauzettel, Phys. Rev. Lett. 94, 166803 (2005).

[18] K. A. Matveev, Phys. Rev. B 51, 1743 (1995).

[19] A. O. Gogolin and A. Komnik, Phys. Rev. B 73, 195301 (2006).

[20] S. Ghoshal and A. B. Zamolodchikov, Int. J. Mod. Phys. A, 9, 3841 (1994).

[21] P. Fendley, A. W. W. Ludwig, and H. Saleur, Phys. Rev. B 52, 8934 (1995).

[22] P. Fendley, A. W. W. Ludwig, and H. Saleur, Phys. Rev. Lett. 74, 3005 (1995).

[23] P. Fendley and H. Saleur, Phys. Rev. B 54, 10845 (1996).

[24] A. Komnik and H. Saleur, Phys. Rev. Lett. 96, 216406 (2006).

[25] H. Saleur and U. Weiss, Phys. Rev. B 63, 201302R (2001).

[26] L. S. Levitov and M. Reznikov, Phys. Rev. B 70, 115305 (2004).

[27] H. Baur, A. Fubini, and U. Weiss, Phys. Rev. B 70, 024302 (2004).

[28] U. Weiss, Quantum Dissipative Systems, 2nd ed. (World Scientific, Singapore, 1999). 
[29] A. Komnik and R. Egger, Phys. Rev. Lett. 80, 2881 (1998).

[30] J. Kim, K. Kang, J.-O. Lee, K.-H. Yoo, J.-R. Kim, J. W. Park, H. M. So, and J. J. Kim, J. Phys. Soc. Jpn. 70, 1464 (2001).

[31] B. Gao, A. Komnik, R. Egger, D. C. Glattli, and A. Bachtold, Phys. Rev. Lett. 92, 216804 (2004).

[32] B. Trauzettel, R. Egger, and H. Grabert, Phys. Rev. Lett. 88, 116401 (2002). 